\title{
Psychiatric disturbances in patients undergoing open-heart surgery
}

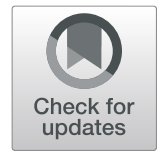

\author{
Osama Younes ${ }^{1 *}$ (D), Reham Amer ${ }^{1}$, Hosam Fawzy ${ }^{2}$ and Gamal Shama ${ }^{1}$
}

\begin{abstract}
Background: Emotional and behavioral problems have been noted in a considerable number of patients after open-heart surgery. However, great discrepancy exists in the literature regarding the frequency and the course of psychiatric symptoms, cognitive performance, and quality of life among those patients. This prospective study was designed to assess the pre- and postoperative psychiatric profile, as well as the quality of life of patients undergoing open-heart surgery.

Methods: One hundred patients who were prepared for cardiac surgery and met our selection criteria were recruited in this study. Each patient was subjected to the Hospital Anxiety and Depression Scale, the Mini-Mental State Examination with selective subtests of Wechsler Adult intelligence scale, and the Short Form 36 questionnaire to assess psychiatric symptoms, cognitive performance, and quality of life respectively. Assessment was done for each of the evaluated items before surgery as well as at 1 week and 6 months postoperatively.

Results: The anxiety and depressive symptoms were significantly lower at 6 months postoperatively than preoperatively. The cognitive performance declined after 1 week, then improved significantly at the 6 -month follow-up. The quality of life scale was significantly lower preoperatively than after surgery.

Conclusions: Anxiety and depressive symptoms, which occurred in substantial percentage of patients undergoing open-heart surgery, were gradually improved with time. Cognitive functions showed early deterioration with significant improvement at 6 months. Psychiatric problems had an adverse impact on patients' quality of life which raised the importance of psychiatric consultation before and after cardiac surgeries to shorten recovery time.
\end{abstract}

Keywords: Cardiac surgery, Cognitive impairment, Psychiatric complications, Quality of life

\section{Background}

Postoperative psychiatric disorders and cognitive dysfunction are among the important causes of morbidity and mortality following cardiac surgery [1]. After coronary artery bypass grafting (CABG) surgery, up to $60 \%$ of patients experienced depressive symptoms, and about $23 \%$ of patients suffer from major depression that are usually associated with poor quality of life and degeneration of venous grafts [2]. Additionally, anxiety symptoms were documented in approximately $40 \%$ of patients before cardiac surgery with expected gradual improvement few weeks after surgery [3]. However, the time and the course of postoperative anxiety and depression remain a source of considerable debate $[4,5]$.

\footnotetext{
* Correspondence: osamaebrahim2012@gmail.com

${ }^{1}$ Department of Psychiatry and Neurology, Faculty of Medicine, Tanta

University, Tanta 31527, Egypt

Full list of author information is available at the end of the article
}

Postoperative cognitive decline is another troublesome issue after cardiac surgery. Some studies showed that CABG patients had worse cognitive profile at baseline, with gradual improvement over a period of 1 year [6]. On the other hand, other studies [7] established an early improvement in cognitive performance among CABG patients followed by late cognitive decline. Van den Goor et al. [8] reported that uncomplicated open-heart surgery has no harmful effect on cognitive functions. Assessment of time, course, and severity of cognitive decline after CABG surgery is crucial for early application of neuroprotective approaches [9].

Despite important advances in techniques for anesthesia and cardiac operations, open-heart surgery remains a major life event that has an important impact on the patients and their families [1]. Nowadays, the surgical treatment of heart diseases is aiming not 
only to alleviate patients' symptoms but also to improve quality of life (QOL) and increased survival rate after surgery [10].

The objective of this prospective study is to evaluate the psychiatric symptoms, cognitive profile, and QOL before and after cardiac surgery in 100 patients undergoing open-heart surgery with short-term follow-up. Additionally, the study assessed the different predictors affecting the above-mentioned items.

\section{Methods}

This prospective study was carried out at Neuropsychiatry and Cardiothoracic Surgery Departments in Tanta University Hospital from October 2016 to November 2017 after approval of the institutional ethical committee. One hundred patients, who were prepared for openheart surgery, were recruited in this study. Inclusion criteria involved cases between 18 and 65 years who have completed more than 6 years of education (to be able to participate in the psychological evaluation). Patients were excluded if they did not meet the inclusion criteria or if they suffered from any history of previous/current psychiatric, neurological, and/or serious metabolic disorders that could affect their cognitive functions.

After obtaining an informed consent, all patients were subjected to full psychiatric history, mental state examination, and physical and neurological evaluations. Laboratory works (complete blood count, serum urea, serum creatinine level, liver function test) were done preoperatively to exclude patients who have serious metabolic disorders that might affect the sensorium.

Psychiatric assessment included Hospital Anxiety and Depression Scale (HADS) and psychometric tests. Each item was evaluated preoperatively (within $48 \mathrm{~h}$ prior to surgery) and postoperatively (at 1 week and 6 months after operation). The Arabic form of HADS was used to identify and rate symptoms of anxiety and depression $[11,12]$. Comprehensive psychometric tests were done for measuring global cognition using the Arabic form of the Mini-Mental State Examination (MMSE) [13, 14]. The cognitive domains (attention/psychomotor speed, verbal memory, and visuospatial memory) were assessed by selective subtests of Wechsler Adult Intelligence Scale (WAIS-IV) "Digit span, Arithmetic, Digit symbol and Block design tests" in its Arabic form [15]. The Arabic form of the Short Form 36 questionnaire (SF-36) was used for quantitative assessment of health-related quality of life (HRQOL) [16, 17]. The SF-36 questionnaire yields an eight-scale multi-item profile of physical and mental health domains (general health, bodily pain, physical functioning, role physical, vitality, social functioning, and role emotional and mental health).

Additionally, the effect of certain patient-related factors (age, sex, habits, and comorbidities) and surgery- related factors (type of surgery, ischemic time, intensive care unit-length of stay, total bypass time and postoperative delirium) on outcomes were also analyzed.

\section{Statistical analysis}

Data were analyzed using Statistical Program for Social Science (SPSS) version 20.0, IBM, Armonk, NY, USA. Quantitative data were expressed as mean \pm standard deviation (SD). Qualitative data were expressed as frequency and percentage. A one-way analysis of variance (ANOVA) was used for comparison among different time points. Pearson's correlation coefficient $(r)$ test was used for correlating data.

\section{Results}

Of the total participants $(n=100)$ in the study (Table 1), 54 (54\%) were male and $46(46 \%)$ were female. The mean age of this study was $46.7 \pm 12.1$ years. Most of the patients (83\%) were smoker, and $17 \%$ were non-smoker. Regarding preoperative comorbidities in the studied patients, 31\% were hypertensive and $13 \%$ were diabetic. Seventy patients (70\%) undergone valve replacement surgery, 27 (27\%) had CABG, and 3 (3\%) had combined CABG and valve surgery. During the surgery, the mean intra-operative ischemic time was $90.23 \pm 28.94 \mathrm{~min}$, and the mean intra-operative total bypass time was $132.45 \pm 35.04 \mathrm{~min}$. The mean intensive care unitlength of stay (ICU-LOS) after surgery was $4.36 \pm$ 3.26 days, and the number of patients who had postoperative delirium was 22 .

Regarding the incidence of anxiety symptoms, $30 \%$ of patients had preoperative anxiety symptoms, decreased to $12 \%$ at 1 week after surgery, and reached to $6 \%$ at 6 months after surgery. The depressive symptoms were encountered among $18 \%$ of the patients at the preoperative records, which decreased after surgery to $9 \%$ and $6 \%$ at 1 week and 6 months respectively.

Table 1 Demographic and patients' characteristics in the study

\begin{tabular}{llll}
\hline Demographic data & Age & Range & $22-65$ \\
& & Mean \pm SD & $46.7 \pm 12.1$ \\
& Sex & Male & $54(54 \%)$ \\
& & Female & $46(46 \%)$ \\
& Special habits & Smoker & $83(83 \%)$ \\
& & Non-smoker & $17(17 \%)$ \\
Clinical comorbidity & Hypertension & $31(31 \%)$ \\
& Diabetes mellitus & $13(13 \%)$ \\
& Previous cardiac surgery & $5(5 \%)$ \\
Type of surgery & Coronary artery bypass surgery & $27(27 \%)$ \\
& Valve surgery & $70(70 \%)$ \\
& Combined & $3(3 \%)$ \\
\hline
\end{tabular}


When the mean scale values of anxiety were compared through the three phases, there was a statistically significant improvement $(P<0.001)$ in these symptoms at the final records as compared to preoperative values (Table 2). However, most of this improvement occurred at the first postoperative week $(P<0.006)$. As regards depressive symptoms, there was a statistically insignificant improvement $(P<0.208)$ in the mean values of these symptoms at 1 week after surgery, as compared to preoperative values, with a statistically significant improvement $(P<0.012)$ at 6 -month record (Table 2$)$.

As regards cognitive performance, there was an insignificant decrease in MMSE score at 1 week postoperatively $(P<0.238)$, which began to improve after 6 months without statistically significant difference $(P<$ 0.774). The mean values of Digit span, Arithmetic, Digit symbol and Block design tests were significantly lower 1 week after surgery than both preoperatively and after 6 months (Table 2).

The mean value of SF36 showed a statistically significant difference through the different record times. The mean of the eight domains of SF36 was lower preoperatively than 1 week after surgery and after 6 months (Fig. 1).

Regarding the effect of different variables on the outcomes (Table 3), age and diabetes mellitus had a statistically significant inverse correlation with anxiety and depressive symptoms. Additionally, hypertension, age, total bypass time, ischemic time, and delirium were significant factors predicting cognitive performance with a statistically significant inverse correlation. The diabetes mellitus and ICUL-LOS had a statistically significant inverse correlation with mental and physical HRQOL (Table 3). Anxiety and depression scales had significant inverse correlations with both physical and mental components of HRQOL (Table 4). Global cognitive functions, as monitored by MMMS, were significantly correlated with both components of the SF-36 questionnaire (Table 4).

\section{Discussion}

Nowadays, surgeons centered their attention on the physical and mental recovery after operations [5]. Various studies [18] have reported that psychiatric problems, particularly depression and anxiety, occur in proportions of patients undergoing cardiovascular surgery.

We reported that anxiety scales showed a significant improvement at the first postoperatively week compared to the preoperative baseline values and continued to improve, though insignificantly, thereafter. Depressive symptoms were statistically significantly improved after 6 months postoperatively when compared to the preoperative value. These results were coinciding with the results of Hoyer et al. [4] who found that anxiety and depressive symptoms decreased 6 weeks after surgery and remained stable at 6-month follow-up. Petersen et al. [5] reported comparable results at 6-month follow-up. Conversely, at the first postoperative week, they reported that anxiety symptoms were not changed significantly from the preoperative value, and depression increased significantly when compared with their preoperative baseline values. These debates in the findings of Petersen et al. [5] may be attributed to their inclusion of older age population (mean age 62 years), which may reflect the effect of age-related changes and the duration of preoperative admission with different timing in obtaining their preoperative value (up to 1 week before surgery). Hernandez-Palazon et al. [19] emphasized that lengthy preoperative hospital stay demonstrated a greater anxiety score among patients undergoing CABG.

In the current study, $30 \%$ of patients had preoperative anxiety symptoms and $18 \%$ of patients had preoperative depressive symptoms. This incidence was in the middle of the spectrum reported in the literature for these symptoms that varies from 20 to $35 \%$ for anxiety and from 8 to $47 \%$ for depression [2, 20]. This wide range among different series could be explained by the use of different methods of diagnosis, several scales for

Table 2 Mean value of psychiatric symptoms and cognitive performance at different time points in the study

\begin{tabular}{llllllllll}
\hline & & Preoperative & After 1 week & After 6 months & $F$ & $P$ & P1 & P2 & P3 \\
\hline HADS scale & Anxiety & $7.99 \pm 4.2$ & $6.55 \pm 3.5$ & $5.92 \pm 2.8$ & 8.453 & $0.001^{*}$ & $0.006^{*}$ & 0.238 & $0.001^{*}$ \\
& Depression & $7.3 \pm 3.3$ & $6.5 \pm 3.2$ & $5.9 \pm 3.1$ & 4.699 & $0.017^{*}$ & 0.208 & 0.430 & $0.012^{*}$ \\
\multirow{2}{*}{ Mini-Mental State Examination } & $25.70 \pm 1.46$ & $25.55 \pm 1.81$ & $25.74 \pm 1.613$ & 1.162 & 0.316 & 0.238 & 0.219 & 0.774 \\
WAIS-IV subtests & Digit symbol test & $38.78 \pm 13.71$ & $33.44 \pm 13.6$ & $40.46 \pm 14.02$ & 12.143 & $0.001^{*}$ & $0.006^{*}$ & $0.001^{*}$ & 0.391 \\
& Forward span & $7.67 \pm 2.29$ & $6.99 \pm 2.33$ & $7.83 \pm 2.57$ & 4.51 & $0.026^{*}$ & $0.014^{*}$ & $0.006^{*}$ & 0.574 \\
& Backward Span & $5.43 \pm 2.26$ & $4.71 \pm 2.21$ & $5.81 \pm 2.38$ & 5.621 & $0.007^{*}$ & $0.024^{*}$ & $0.003^{*}$ & 0.248 \\
& Block design test & $26.11 \pm 8.707$ & $21.58 \pm 6.4$ & $28.18 \pm 9.171$ & 11.32 & $0.001^{*}$ & $0.001^{*}$ & $0.001^{*}$ & 0.104 \\
& Arithmetic test & $7.98 \pm 1.583$ & $7.24 \pm 1.585$ & $7.99 \pm 1.678$ & 5.687 & $0.012^{*}$ & $0.006^{*}$ & $0.005^{*}$ & 0.875 \\
\hline
\end{tabular}

$F$ for one-way ANOVA (analysis of variance), $P 1$ comparing preoperative and after 1 -week scales, $P 2$ comparing one week and after 6 -month scales, $P 3$ comparing preoperative and after 6 months postoperatively

*Statistically significant at $P<0.05$ 


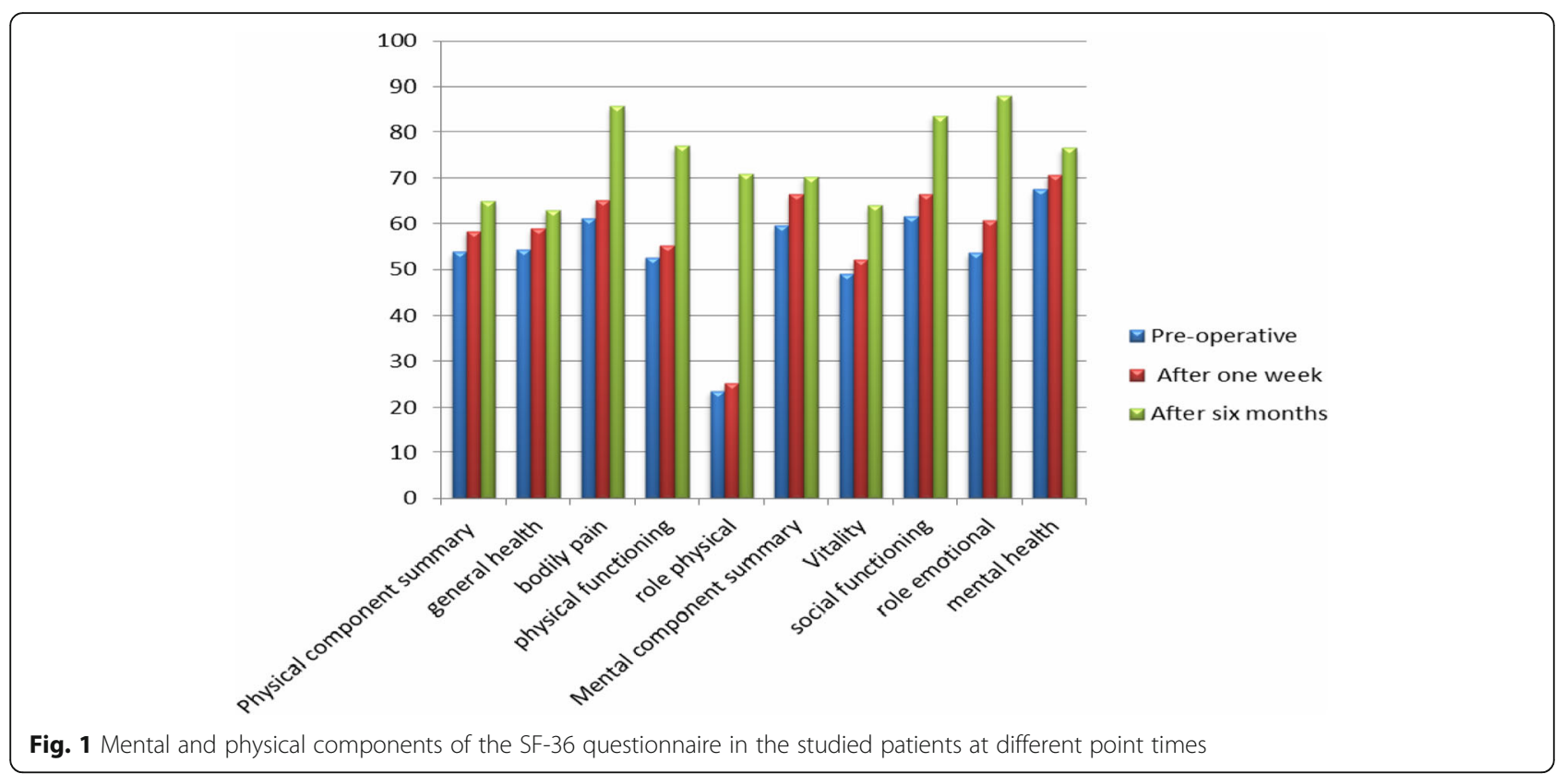

symptom evaluation, and by the variation in selection criteria and patient characteristics among studies.

As regards factors predicting anxiety and depressive symptoms, in this research, there was no impact of gender on HADS, which agree with the results of Hernandez-Palazon et al. [19]. In contrast, Koivula et al. [21] observed a higher preoperative anxiety level in women prepared for cardiac surgery. The reason for this variation may be explained by hormonal fluctuation in women. In our work, there was no influence of smoking on HADS scores, and this result was in the same line with Thomsen et al. [22] who found that smoking might

Table 3 Stepwise linear regression analysis for significant variables affecting final outcomes

\begin{tabular}{llll}
\hline & Variable & OR $(95 \%$ Cl) & $P$ value \\
\hline HADS depression & Age & $0.542(0.291-0.954)$ & $0.012^{*}$ \\
& Diabetes mellitus & $0.505(0.341-0.819)$ & $0.041^{*}$ \\
HADS anxiety & Age & $0.569(0.431-0.830)$ & $0.024^{*}$ \\
& Diabetes mellitus & $0.621(0.258-0.921)$ & $0.039^{*}$ \\
Cognition (MMSE) & Hypertension & $0.546(0.205-0.743)$ & $0.043^{*}$ \\
& Age & $2.417(1.544-6.541)$ & $0.014^{*}$ \\
& Total bypass time & $2.621(1.258-8.921)$ & $0.029^{*}$ \\
& Ischemic time & $2.418(1.574-9.623)$ & $0.041 *$ \\
SF-36 (physical) & Delirium & $2.541(1.974-7.519)$ & $0.001 *$ \\
& ICU-LOS & $1.423(1.326-4.203)$ & $0.001 *$ \\
SF-36 (mental) & Diabetes mellitus & $0.515(0.146-0.829)$ & $0.017 *$ \\
& ICU-LOS & $1.631(1.203-2.691)$ & $0.002^{*}$ \\
& Diabetes mellitus & $1.853(1.307-7.517)$ & $0.044^{*}$ \\
\hline
\end{tabular}

*Statistically significant at $P<0.05$ affect the wound-healing process without improving anxiety. A significant correlation between HADS Scale and ICU-stay was not observed in our study that coincided with the work of Poole et al. [23]. In contrast, Milton et al. [24] documented a positive relationship between symptoms of anxiety and depression with the length of ICU stay, but the latter study was on all ICU patients and not only patients with open-heart surgery. In the current study, elderly patients and those with comorbid diabetes mellitus had greater degrees of anxiety and depression. Eng et al. [25] also documented that patients, who had comorbid diseases, were more prone to psychiatric symptoms which could be attributed to the additional burden of these comorbidities on their health. Aging and comorbid diseases might interrupt the daily activities of the patients and caused them to feel easily fatigued, restless, anxious, and more depressed.

Cardiac patients usually have cardiovascular disease and/or systemic hypertension, which can reduce blood flow to the brain, increase the incidence of brain damage, and affect cognition. Accordingly, it is important to

Table 4 The impact of psychiatric disturbances on HRQOL in the study

\begin{tabular}{|c|c|c|c|c|c|}
\hline \multicolumn{2}{|c|}{ Variables } & \multicolumn{4}{|l|}{ SF-36 } \\
\hline & & \multicolumn{2}{|l|}{ Physical } & \multicolumn{2}{|l|}{ Mental } \\
\hline & & $R$ & $P$ & $R$ & $P$ \\
\hline \multirow[t]{2}{*}{ HADS } & Depression & -0.493 & $0.010^{*}$ & -0.745 & $0.001^{*}$ \\
\hline & Anxiety & -0.428 & $0.015^{*}$ & -0.695 & $0.001^{*}$ \\
\hline \multicolumn{2}{|c|}{ Cognition (MMSE) } & 0.512 & $0.008^{*}$ & 0.812 & $0.001^{*}$ \\
\hline
\end{tabular}

*Statistically significant at $P<0.05$ 
evaluate cognitive performance in the patients before and after open-heart operations [26]. The current study observed a significant postoperative cognitive decline (POCD) in the studied neuropsychological tests (for attention, verbal memory, and visuospatial memory) during the first postoperative week that improved significantly at the 6-month follow-up. These results were in the same line of Bruce et al. [27] who documented that $44 \%$ of CABG patients achieved poor results in cognitive assessment in the first week after surgery, and they recovered to their preoperative levels by 8 weeks. They explained the POCD by the effects of mood state, the recovery from anesthesia, and the postoperative pain. Bokeriia et al. [28] found that about 40\% of patients had short-term POCD in the first weeks after cardiac surgery, usually improving few months after discharge. They proposed that the microembolic load at the left and right middle cerebral artery associated with a verbal and nonverbal memory declines respectively.

On the contrary, Knippa et al. [29] found that memory impairment and decreased verbal learning after CABG can persist for months. Newman et al. [30] documented early improvement in cognitive performance in CABG patients followed by a later cognitive decline (incidence of POCD was $24 \%$ at 6 months after operation, and increased to $42 \%$ at 5 years). These divergent findings may be related to the longer duration of follow-up or due to the older age of patients of the later studies (mean age in Newman et al. study was 61 years and in Knippa et al. was 67 years) that may be associated with involution changes in brain structure and function, which may affect cognition.

As regards predictors affecting cognitive functions, this research found no significant differences in cognitive performance between male and female patients and also between smokers and non-smokers. These results were in the same line with many of previous studies [26, 31, 32]. In our work, we found no role for ICU-LOS on cognition as documented also by Harrington et al. [33]. However, we observed that hypertension, age, total bypass time, ischemic time, and delirium were strong predictors of cognitive performance and had a statistically significant inverse correlation with it. Glance et al. [34] and Tarakji et al. [35] also reported a strong association between cognitive impairment and comorbid hypertension. Systemic hypertension may lead to structural and functional cerebro-vascular changes responsible for the cognitive deficits. Conversely, in the study of Cormack et al. [36], hypertension was not a significant predictor of cognitive outcome. Many of previous studies $[28,37]$ agree with our findings that postoperative verbal memory decline was correlated with length of bypass time. In contrast to our study,
Nguyen et al. [31] observed no differences in cognitive functions between the delirious and non-delirious subjects after 6 to 9 months. This dissimilarity in the reported severity and type of cognitive dysfunction between different studies may be attributed to the difference in: methodology, study population characteristics, the selected definition of cognitive impairment, and the time of postoperative evaluation.

Quality of life is progressively being evaluated as a crucial outcome in patients after open-heart surgery. In our study, the HRQOL had a significant improvement $(p<$ 0.05 ) in the first postoperative week, and this improvement was well maintained at the sixth postoperative month. This is in agreement with Sibilitz et al. [38] who also documented marked improvement on HRQOL at 6-month follow-up after heart valve surgery. Petersen et al. [5] reported initially significant poor outcomes in HRQOL at the first postoperative week, with gradual improvement up to 6 months after surgery. Many other studies [36, 37] reported up to $26.8 \%$ and $58.4 \%$ nonimprovement rates in physical HRQOL and mental HRQOL respectively within 2 years after coronary intervention. The variation in the results may be explained by the age difference between studies, the application of different methods for assessing QOL, the use of various techniques for statistical analysis, and the dissimilar criteria for classifying patients as improved, remaining stable, or deteriorated. The study of Middel et al. [39] found a decline in HRQOL 6 months after CABG surgery, and they explained their results by the influence of psychiatric disorders and type D personality (negative affectivity and social inhibition) that considered crucial factors for poor outcomes. All these variables were excluded in our study, which could explain our superior results.

When studying predictors affecting HRQOL, the current study established that mental and physical HRQOL was not affected by gender difference and this was in the same line with Diab et al. [40]. On the contrary, Le Grande et al. [41] reported that female had a decline in postoperative QOL and explained that with poorer emotional state that may affect mental HRQOL. Kurlansky et al. [42] found that male sex was correlated with worse HRQOL, but their patients were around 80 years and were undergone isolated CABG. Additionally, our work found no role for cigarette smoking on mental and physical HRQOL as documented also by Hein et al. [43]. Lindsay et al. [44] had an opposite view as they found that smoking affects the HRQOL significantly at long-term follow-up and they deemed that progression of atherosclerosis, particularly in vein grafts, is the cause of their finding.

The ICU-LOS and diabetes mellitus were the significant predictors of mental and physical QOL in our work 
that had a statistically significant inverse correlation with it. Kurlansky et al. [42] had the same opinion as they found that diabetes mellitus and postoperative ICU treatment $>3$ days and cardiac symptoms $>120$ days before the operation have been associated with poor HRQOL. Le Grande et al. [41] added that certain diseases (as myocardial infarction, atrial fibrillation, chronic heart failure), some demographic factors (as work status), and psychological distress could affect the HQOL. Welke and colleagues [45] found that high body mass index $>35 \mathrm{~kg} / \mathrm{m}^{2}$, comorbid chronic obstructive pulmonary disease, and peripheral vascular disease were related to poor HRQOL after CABG, but these variables were not studied in our research.

The current study supports previous findings $[39,46,47]$ that anxiety and depressive symptoms had an adverse impact on physical and mental HRQOL. Furthermore, the decline in cognitive performance of the patients was also associated with poorer HRQOL scores. These findings raise the importance of psychiatric consultation before and after cardiac surgeries to improve functions and shorten recovery time.

\section{Limitations}

The main shortcomings of this study are the absence of a control group and the short-term follow-up. Future studies would need to replicate the findings of this work with a larger sample size and with a long-term follow-up to understand the changes in psychiatric profile and cognition performance following heart surgery. Also, comparing the postoperative psychiatric/cognitive profiles of cardiac surgery patients with group of patients receiving prophylactic psychiatric therapy may aid in evaluating the progression of mental function after surgery.

\section{Conclusions}

Anxiety and depressive symptoms, which occurred in $30 \%$ and $18 \%$ respectively of our patients, had an adverse impact on HRQOL and were gradually improved with time. However, cognitive functions showed early decline followed by improvement at 6 months. These findings raised the importance of psychiatric consultation, pharmacotherapy, and cognitive therapy before and after cardiac surgeries to improve surgical outcomes and to enhance patients' HRQOL. A team of psychiatrists with cardiac surgeons should work together to prevent any psychiatric complications that might associate cardiac surgery.

\footnotetext{
Abbreviations

CABG: Coronary artery bypass grafting; HADS: Hospital anxiety and depression scale; HRQOL: Health-related quality of life; ICU-LOS: Intensive care unit-length of stay; MMSE: Mini-Mental State Examination;

POCD: Postoperative cognitive decline; QOL: Quality of life; SF-36: Short Form 36 questionnaire; SPSS: Statistical Program for Social Science; WAIS-

IV: Wechsler Adult Intelligence Scale
}

\section{Acknowledgements}

We would like to express our deep appreciation to Dr. Ehab Ramadan Professor of the Neuropsychiatry Department, University of Tanta, Egypt, for his valuable advice throughout the period of the work. We also express our gratitude to the whole medical staff in the Cardiothoracic and Neuropsychiatry departments at Tanta University Hospitals who shared in managing and following-up the patients. Many thanks also to the clinical psychologists, who helped in the psychometric evaluation for the recruited patients.

\section{Authors' contributions}

All authors certify that the submission to this work is original and that they have reviewed and confirmed the accuracy of the whole manuscript and have approved the final submitted version. OY contributed to the collection and interpretation of data, statistical analysis of the results, drafting of the work, and writing of the manuscript. RA contributed to the design of the work, interpretation of the data, review of the results' analysis, and sharing in the writing, revising, and editing this manuscript. HF contributed to the design of the work, selection of the recruited subjects, interpretation of the data, and revision of the manuscript. GS contributed to the conception and design of the work, interpretation of the data, editing, and critical revision of the manuscript

\section{Funding}

This study was self-funded. No funds were received.

\section{Availability of data and materials}

The datasets used and analyzed during the current study are available from the corresponding author on reasonable request.

\section{Ethics approval and consent to participate}

The study was approved by the Ethical Research Committee of the Faculty of Medicine, Tanta University. An informed consent was obtained from all participants to be included in this study.

\section{Consent for publication}

Each of the participants was given a code number, and his anonymity was preserved (his individual person's data was omitted and not included in the submitted manuscript).

\section{Competing interests}

The authors declare that they have no competing interests.

\section{Author details}

${ }^{1}$ Department of Psychiatry and Neurology, Faculty of Medicine, Tanta University, Tanta 31527, Egypt. ${ }^{2}$ Cardio-Thoracic Surgery Department, Faculty of Medicine, Tanta University, Tanta, Egypt.

Received: 10 July 2019 Accepted: 9 August 2019

Published online: 17 September 2019

\section{References}

1. Kadoi Y, Goto F (2006) Factors associated with postoperative cognitive dysfunction in patients undergoing cardiac surgery. Surg Today 36(12): 1053-1057

2. Mendonca da Cunha DM, Dos Anjos TS, Franca Lisboa GC, Tavares de Mattos MC, Vale CL, de CJ et al (2016) Depressive symptoms in patients with coronary artery disease. Invest Educ Enferm 34(2):323-328

3. Oxlad M, Wade TD (2008) Longitudinal risk factors for adverse psychological functioning six months after coronary artery bypass graft surgery. J Health Psychol 13(1):79-92

4. Hoyer J, Eifert GH, Einsle F, Zimmermann K, Krauss S, Knaut M et al (2008) Heart-focused anxiety before and after cardiac surgery. J Psychosom Res 64(3):291-297

5. Petersen J, Vettorazzi E, Winter L, Schmied W, Kindermann I, Schafers HJ (2016) Physical and mental recovery after conventional aortic valve surgery. J Thorac Cardiovasc Surg 152(6):1549-1556

6. McKhann GM, Grega MA, Borowicz LM Jr, Bechamps M, Selnes OA, Baumgartner WA et al (2002) Encephalopathy and stroke after coronary artery bypass grafting: incidence, consequences, and prediction. Arch Neurol 59(9):1422-1428 
7. Newman MF, Grocott HP, Mathew JP, White WD, Landolfo K, Reves JG et al (2001) Report of the substudy assessing the impact of neurocognitive function on quality of life 5 years after cardiac surgery. Stroke 32(12):28742881

8. Van den Goor J, Saxby B, Tijssen J, Wesnes K, De Mol B, Nieuwland R (2008) Improvement of cognitive test performance in patients undergoing primary CABG and other CPB-assisted cardiac procedures. Perfusion 23:267-273

9. Selnes OA, Grega MA, Bailey MM, Pham L, Zeger S, Baumgartner WA et al (2007) Neurocognitive outcomes 3 years after coronary artery bypass graft surgery: a controlled study. Ann Thorac Surg 84(6):1885-1896

10. Bradt J, Dileo C, Magill L, Teague A (2016) Music interventions for improving psychological and physical outcomes in cancer patients. Cochrane Database Syst Rev (8):CD006911

11. El-Rufaie OE, Absood G (1987) Validity study of the Hospital Anxiety and Depression Scale among a group of Saudi patients. Br J Psychiatry 151:687-688

12. Zigmond AS, Snaith RP (1983) The hospital anxiety and depression scale. Acta Psychiatr Scand 67(6):361-370

13. Albanna M, Yehya A, Khairi A, Dafeeah E, Elhadi A, Rezgui L et al (2017) Validation and cultural adaptation of the Arabic versions of the MiniMental Status Examination - 2 and Mini-Cog test. Neuropsychiatr Dis Treat 13:793-801

14. Reid MC, Maciejewski PK, Hawkins KA, Bogardus ST Jr (2002) Relationship between alcohol consumption and Folstein mini-mental status examination scores among older cognitively impaired adults. J Geriatr Psychiatry Neurol 15(1):31-37

15. Abou-Mrad F, Chelune G, Zamrini E, Tarabey L, Hayek M, Fadel P (2017) Screening for dementia in Arabic: normative data from an elderly Lebanese sample. Clin Neuropsychol. 31(sup1):1-19. https://doi.org/10.1080/13854 046.2017.1288270 Epub 2017 Feb 23

16. Al-Ghamdi MS, Taha AZ, Ahmad B, Khalil MS (2002) Quality of life in a sample of hypertensive patients attending primary health care facilities in Al-khobar, Saudi Arabia. J Family Community Med 9(1):25-32

17. Garratt AM, Ruta DA, Abdalla MI, Russell IT (1994) SF 36 health survey questionnaire: II. Responsiveness to changes in health status in four common clinical conditions. Qual Health Care 3(4):186-192

18. Connerney I, Shapiro PA, McLaughlin JS, Bagiella E, Sloan RP (2001) Relation between depression after coronary artery bypass surgery and 12-month outcome: a prospective study. Lancet 358(9295):1766-1771

19. Hernández-Palazón J, Fuentes-García D, Falcón-Araña L, Roca-Calvo MJ, Burguillos-López S, Doménech-Asensi P et al (2018) Assessment of preoperative anxiety in cardiac surgery patients lacking a history of anxiety: contributing factors and postoperative morbidity. J Cardiothorac Vasc Anesth. 32(1):236-244

20. Nunes JK, Figueiredo Neto JA, Sousa RM, Costa VL, Silva FM, Hora AF et al (2013) Depression after CABG: a prospective study. Rev Bras Cir Cardiovasc 28(4):491-497

21. Koivula M, Paunonen-IImonen M, Tarkka MT, Tarkka M, Laippala P (2001) Fear and anxiety in patients awaiting coronary artery bypass grafting. Heart Lung 30(4):302-311

22. Thomsen T, Villebro N, Moller AM (2010) Interventions for preoperative smoking cessation. Cochrane Database Syst Rev (7):CD002294

23. Poole L, Kidd T, Leigh E, Ronaldson A, Jahangiri M, Steptoe A (2015) Psychological distress and intensive care unit stay after cardiac surgery: the role of illness concern. Health Psychol 34(3):283-287

24. Milton A, Bruck E, Schandl A, Bottai M, Sackey P (2017) Early psychological screening of intensive care unit survivors: a prospective cohort study. Crit Care 21(1):273

25. Eng HS, Yean LC, Das S, Letchmi S, Yee KS, Bakar RA et al (2011) Anxiety and depression in patients with coronary heart disease: a study in a tertiary hospital. Iran J Med Sci 36(3):201-206

26. Barstow C, McDivitt JD (2017) Cardiovascular disease update: care of patients after coronary artery bypass graft. FP Essent 454:29-33

27. Bruce KM, Yelland GW, Smith JA, Robinson SR (2013) Recovery of cognitive function after coronary artery bypass graft operations. Ann Thorac Surg 95(4):1306-1313

28. Bokeriia LA, Golukhova EZ, Breskina NY, Polunina AG, Davydov DM Begachev AV et al (2007) Asymmetric cerebral embolic load and postoperative cognitive dysfunction in cardiac surgery. Cerebrovasc Dis 23(1):50-56

29. Knipp SC, Matatko N, Wilhelm H, Schlamann M, Massoudy P, Forsting M et al (2004) Evaluation of brain injury after coronary artery bypass grafting. A prospective study using neuropsychological assessment and diffusion-weighted magnetic resonance imaging. Eur J Cardiothorac Surg 25(5):791-800

30. Newman MF, Kirchner JL, Phillips-Bute B, Gaver V, Grocott H, Jones RH et al (2001) Longitudinal assessment of neurocognitive function after coronaryartery bypass surgery. N Engl J Med 344(6):395-402

31. Nguyen Q, Uminski K, Hiebert BM, Tangri N, Arora RC (2018) Midterm outcomes after postoperative delirium on cognition and mood in patients after cardiac surgery. J Thorac Cardiovasc Surg 155(2):660-667.e2

32. Pacini D, Di ML, Leone A, Tonon C, Pettinato C, Fonti C et al (2010) Cerebral functions and metabolism after antegrade selective cerebral perfusion in aortic arch surgery. Eur J Cardiothorac Surg 37(6):1322-1331

33. Harrington MB, Kraft M, Grande LJ, Rudolph JL (2011) Independent association between preoperative cognitive status and discharge location after cardiac surgery. Am J Crit Care 20(2):129-137

34. Glance LG, Wissler R, Mukamel DB, Li Y, Diachun CA, Salloum R et al (2010) Perioperative outcomes among patients with the modified metabolic syndrome who are undergoing noncardiac surgery. Anesthesiology 113(4):859-872

35. Tarakji KG, Sabik JF III, Bhudia SK, Batizy LH, Blackstone EH (2011) Temporal onset, risk factors, and outcomes associated with stroke after coronary artery bypass grafting. JAMA 305(4):381-390

36. Cormack F, Shipolini A, Awad WI, Richardson C, McCormack DJ, Colleoni L et al (2012) A meta-analysis of cognitive outcome following coronary artery bypass graft surgery. Neurosci Biobehav Rev 36(9):2118-2129

37. Hendriksen H, Meulendijks D, Douma TN, Bink DI, Breuer ME, Westphal KG et al (2012) Environmental enrichment has antidepressant-like action without improving learning and memory deficits in olfactory bulbectomized rats. Neuropharmacology 62(1):270-277

38. Sibilitz KL, Berg SK, Thygesen LC, Hansen TB, Kober L, Hassager C et al (2015) High readmission rate after heart valve surgery: a nationwide cohort study. Int J Cardiol 189:96-104

39. Middel B, El BN, Pedersen SS, van Dijk JP, Wynia K, Reijneveld SA (2014) Decline in health-related quality of life 6 months after coronary artery bypass graft surgery: the influence of anxiety, depression, and personality traits. J Cardiovasc Nurs 29(6):544-554

40. Diab M, Bilkhu R, Soppa G, McGale N, Hirani SP, Newman SP et al (2017) Quality of life in relation to length of intensive care unit stay after cardiac surgery. J Cardiothorac Vasc Anesth 31(3):1080-1090

41. Le Grande MR, Elliott PC, Murphy BM, Worcester MU, Higgins RO, Ernest CS et al (2006) Health related quality of life trajectories and predictors following coronary artery bypass surgery. Health Qual Life Outcomes 4:49

42. Kurlansky PA, Williams DB, Traad EA, Zucker M, Ebra G. Eighteen-year followup demonstrates prolonged survival and enhanced quality of life for octogenarians after coronary artery bypass grafting. J Thorac Cardiovasc Surg. 2011;141(2):394-399, 399.e1-3. doi: https://doi.org/10.1016/j.jtcvs.2010. 05.003. Epub 2010 Jun 9

43. Hein OV, Birnbaum J, Wernecke K, England M, Konertz W, Spies C (2006) Prolonged intensive care unit stay in cardiac surgery: risk factors and longterm-survival. Ann Thorac Surg 81(3):880-885

44. Lindsay GM, Hanlon P, Smith LN, Wheatley DJ (2000) Assessment of changes in general health status using the short-form 36 questionnaire 1 year following coronary artery bypass grafting. Eur J Cardiothorac Surg 18(5):557-564

45. Welke KF, Stevens JP, Schults WC, Nelson EC, Beggs VL, Nugent WC (2003) Patient characteristics can predict improvement in functional health after elective coronary artery bypass grafting. Ann Thorac Surg 75(6):1849-1855

46. Lee GA (2009) Determinants of quality of life five years after coronary artery bypass graft surgery. Heart Lung 38(2):91-99

47. Mallik S, Krumholz HM, Lin ZQ, KasI SV, Mattera JA, Roumains SA et al (2005) Patients with depressive symptoms have lower health status benefits after coronary artery bypass surgery. Circulation 111(3):271-277

\section{Publisher's Note}

Springer Nature remains neutral with regard to jurisdictional claims in published maps and institutional affiliations. 